\title{
Criminologie
}

\section{La prison dans la Chine de Mao}

\section{José Rico}

Volume 9, numéro 1-2, 1976

L’emprisonnement au Québec

URI : https://id.erudit.org/iderudit/017059ar

DOI : https://doi.org/10.7202/017059ar

Aller au sommaire du numéro

Éditeur(s)

Les Presses de l'Université de Montréal

ISSN

0316-0041 (imprimé)

1492-1367 (numérique)

Découvrir la revue

Citer ce compte rendu

Rico, J. (1976). Compte rendu de [La prison dans la Chine de Mao]. Criminologie, 9(1-2), 219-231. https://doi.org/10.7202/017059ar d'utilisation que vous pouvez consulter en ligne.

https://apropos.erudit.org/fr/usagers/politique-dutilisation/ 


\section{LA PRISON DANS \\ LA CHINE DE MAO}

José Rico

La plupart des pays occidentaux possèdent et publient, avec une certaine régularité, des renseignements plus ou moins fiables concernant la nature et l'étendue de leur criminalité. La littérature carcérale, elle aussi fort abondante, s'y est par ailleurs enrichie depuis quelques années grâce aux rapports des commissions d'enquête créées à la suite de divers événements survenus récemment dans les prisons de quelques pays (émeutes, prises d'otages suivies ou non d'exécution de ceux-ci, grève du personnel pénitentiaire, utilisation de la torture ou de mauvais traitements, etc.) ainsi qu'aux témoignages d'ex-détenus ou d'associations pour la défense de ceux-ci.

Dans les pays à économie socialiste, les données concernant la criminalité et le système pénitentiaire sont, par contre, fort pauvres. Nous ne disposons à l'heure actuelle que de quelques informations plus ou moins valables sur la criminalité en URSS, en Pologne et en Yougoslavie; et, avant Soljenitsyne, nous ignorions pratiquement tout sur les prisons soviétiques.

L'état de nos connaissances en ce qui a trait à la Chine communiste est encore plus limité. Pour certains, la criminalité professionnelle et la délinquance juvénile auraient été balayées de cet immense continent; l'enthousiaste déferlement moral provoqué par la Révolution de 1949 l'aurait purifié des manifestations criminelles. Un des rares criminologues qui ait visité récemment la Chine, le Dr. Georges Heuyer, professeur honoraire à la Faculté de médecine de Paris. confirmait que dans ce vaste pays, il n'y avait plus de vols ni de prostituées, plus de bandes organisées 
ni de blousons noirs'. Ėtant donné qu'aucune publicité n'est donnée par la presse aux infractions, que les jeunes sont encadrés dans des organisations de pionniers et qu'ils sont obligés de se livrer à des travaux agricoles pendant une partie des loisirs et des vacances, les affirmations découlant de ces témoignages semblent logiques. Il convient cependant de souligner, outre le fait qu'aucune statistique criminelle n'est publiée en Chine, qu'il s'agit là d'une certaine catégorie de témoignages : celle de la presque totalité des étrangers - journalistes, écrivains, ministres, diplomates, universitaires, scientifiques, etc. - qui, nantis des mêmes guides et interprètes, effectuent le même itinéraire organisé, logeant dans les mêmes hôtels, visitant les mêmes institutions et usines, rencontrant les mêmes personnes, se voyant offrir les mêmes banquets au cours desquels sont prononcés les mêmes discours, se conformant en somme à un même rituel invariable et irréel qui ne tient ni de la Chine ni de l'Occident, mais d'un univers abstrait conçu spécialement par les bureaucrates maoïstes à l'usage des hôtes étrangers. Il existerait donc en Occident une masse impressionnante de connaissances partielles et d'informations totalement fausses au sujet de la Chine; et dès qu'il s'agit de la criminalité et des organismes chargés de la supprimer ou de la contrôler - police, tribunaux, prisons - la somme d'erreurs devient littéralement débordante. Quelques Occidentaux ont certes été arrêtés par la police, subi un procès et passé quelque temps de réclusion dans les prisons chinoises. Ils ont, par la suite, écrit des livres sur leur expérience. Cependant, presque tous furent dès le début fortement handicapés par le fait capital qu'ils ne parlaient pas chinois, ou très peu. En outre, de par leur condition d'étrangers, ils recevaient invariablement un traitement spécial - cellules privées, rations différentes, isolement par rapport aux prisonniers chinois. Aucun Occidental n'a jamais été autorisé à visiter les camps de travail de la Chine; à plus forte raison, aucun n'a jamais mérité le triste honneur d'être sélectionné pour la «Réforme par le Travail».

Dans ces conditions, deux livres récents donnent tout leur relief à la propagande, aux récits naïfs ou intéressés de ceux qui, prétendant décrire les réalités chinoises, ne montrent en fait que ce théâtre d'ombres mis en scène pour eux par les autorités maoistes. Il s'agit d'Ombres chinoises, du sinologue Simon Leys" et, surtout

I. J. Pinatel (1971): la Société criminogène, Paris, Calmann-Lévy, p. 33.

2. Paris: Union générale d'éditions (Collection 10-18), 1974. 
en ce qui nous concerne, de Prisonnier de Mao, de Jean Pasqualini".

Jean Pasqualini, en chinois Bao Ruo-wang, est un «cas». Né en Chine de père corse et de mère chinoise, ayant gardé la nationalité française mais parlant comme un vrai Chinois et avec le visage d'un Chinois, il obtient à l'École industrielle de Chang-haj un diplôme de technicien spécialiste en machines-outils. Cette formation, ainsi que sa connaissance de l'anglais, l'amena à travailler avec les Marines américains, ce qui prépara la voie à son inculpation pour des activités contrerévolutionnaires et à son emprisonnement en 1957. Il a passé sept ans dans des prisons et des camps chinois avant d'être libéré en 1964 grâce à la reconnaissance de la Chine communiste par la France. D'où l'intérêt de ce témoignage, absolument unique, même s'il ne couvre qu'une période limitée de l'histoire chinoise.

Deux ans après la prise du pouvoir par Mao, commence en Chine la première campagne pour l'élimination des contrerévolutionnaires. Les procès publics des tribunaux populaires - parfois des dizaines par jour - étaient retransmis par haut-parleurs sur les places publiques et les foules réclamaient souvent à grands cris la peine de mort. En 1957, Mao lance la campagne des Cent Fleurs, au cours de laquelle on invite le peuple chinois à exprimer librement ses critiques à l'égard du gouvernement. Les résultats de cette opération poussèrent le régime à lancer une campagne de Rectification, qui se transforma en lutte ouverte contre les éléments bourgeois et conservateurs. C'est à l'occasion de cette dernière campagne que Pasqualini se trouva en prison.

Son récit nous permet d'avoir d'importants renseignements sur le phénomène criminel, sur la procédure pénale et, surtout, sur le système pénitentiaire chinois.

I. La délinquance juvénile continue à poser de sérieux problèmes dans certaines régions de la Chine, notamment à Canton.

Les délinquants se recrutent en général parmi les jeunes citadins qui ont été envoyés aux champs; ne pouvant s'adapter à la vie paysanne ni supporter la perspective d'un exil définitif, ils reviennent clandestinement en ville; mais là, comme ils ne disposent d'aucun moyen de subsistance, leur seule ressource reste le chapardage et la rapine. Souvent ils se rassemblent à plusieurs et forme de petites bandes de vagabonds, sans asile fixe ni emploi régulier. Quand les gens prennent un voleur sur le fait, ils se contentent en général de le passer

3. Paris: Gallimard. 1974. 
à tabac, ceci souvent à la demande de l'intéressé lui-même qui préfère également ce traitement expéditif à une intervention de la police... Éventuellement, s'il s'agit non d'un vagabond, mais d'un individu ayant un emploi fixe, on signale son cas à l'organisme qui l'emploie. Seules les affaires plus sérieuses - vols à main armée, brigandage organisé, meurtres - passent devant les tribunaux et sont punis, suivant leur gravité, soit de mort (par fusillade), soit d'emprisonnement, soit de rehabilitation par le travail".

Aussi, contrairement à ce que croient beaucoup d'étrangers, la délinquance juvénile n'a pas disparu en Chine populaire. Le vol, par exemple, subsiste toujours car l'instinct d'appropriation (par voie légale ou illégale) demeure encore, même s'il ne s'exprime que sur une échelle modeste. Certains objets, tels les montres suisses ou les appareils de photo exercent, surtout parmi la jeunesse, une véritable fascination et sont source pour leurs heureux propriétaires d'une fierté et d'un prestige considérables. Il en est de même des vélos, qui tiennent en Chine la place occupée par les autos dans les sociétés occidentales. Si cela n'existait réellement en Chine, on se demande pourquoi dans les parkings de vélos, payants et gardés, toutes les bicyclettes sont munies d'un cadenas.

Il reste difficile d'évaluer dans quelle mesure les esprits se sont apaisés et soumis en Chine. Toujours est-il que S. Leys rapporte que, durant son dernier séjour de six mois dans ce pays, il a été témoin d'un plus grand nombre de disputes, voire même de rixes, qu'en cinq ans de vie à Hong-Kong, ville beaucoup plus agitée et tendue (p. 78).

Quant à la criminalité adulte, de nombreux prisonniers ont été condamnés pour détournements de fonds, viol, adultère, abandon de foyer et homosexualité. Les délits d'ordre moral ne semblent pas être traités à la légère en Chine, où les déviations par rapport à la norme ne sont pas appréciées, ni considérées comme tolérables. Selon Pasqualini :

Après la révolution, de nombreux chanteurs d'opéra masculins furent poursuivis parce qu'ils incarnaient des temmes. La sodomie et le viol peuvent être punis de mort. Les femmes sont condamnées à cinq ans pour rapports conjugaux préconjugaux ou extra-conjugaux. Un homme marié qui séduit une femme mariée écope de dix ans. Un homme marié qui séduit une femme non mariée recevra une sentence indéterminée mais lourde, et sa partenaire une sentence légère. Le lesbianis- 
me a toujours été rare en Chine, mais l'homosexualité, autrefois très répandue, n'est plus tolérée désormais. J'ai lu des articles sur des hommes qui se faisaient violer dans les prisons occidentales. En Chine, le coupable serait fusillé sur-le-champ (p. 198-199).

Les peines appliquées dans les cas de délits contre la moralité sont particulièrement sévères. Pasqualini cite le cas du coiffeur d'un camp chinois, condamné à sept ans de travaux forcés, déclaré coupable d'avoir séduit en prison un garçon de dix-neuf ans, condamné à mort et exécuté par un gardien devant tous les prisonniers quelques secondes après la lecture de la sentence.

En ce qui concerne les délits politiques, de lourdes peines d'emprisonnement sont prévues contre ceux qui, comme Pasqualini, sont accusés d'avoir travaillé pour des puissances étrangères, de faire obstruction au mouvement coopératif (par exemple, un propriétaire de terres qui les cultivait entièrement avec sa famille sans employer des ouvriers), de s'être rendus coupables de "provocation matérialiste» (ce fut le cas d'un fonctionnaire qui avait, pendant la période des Cent Fleurs, conçu un système pour récompenser les meilleurs rendements individuels par des primes).

II. La procédure pénale chinoise ressemble, dans ses grandes lignes, à celle des pays occidentaux. Si l'on prend comme exemple le cas de Pasqualini : il fut arrêté chez lui par des policiers de la Police de sécurité qui, après avoir procédé à la lecture de l'accusation et lui avoir fait signer le mandat d'arrêt, l'amenèrent, menottes aux poings, au Centre de détention préventive de Pékin pour fins d'interrogatoire.

Contrairement à la pratique des pays occidentaux, la durée de l'interrogatoire peut être excessivement longue (plus de quinze mois dans le cas de Pasqualini). L'inculpé qui résiste à un interrogatoire s'expose aux pires conséquences. Par ailleurs, la police de Mao a perfectionné ses méthodes d'interrogation et a atteint un tel degré de raffinement qu'il est presque impossible de lui résister. Leur but n'est pas tellement de faire que l'inculpé invente des crimes inexistants, mais de lui faire admettre que la vie ordinaire qu'il menait était pourrie, coupable et passible de châtiment, puisqu'elle ne correspondait pas à la conception que le régime a de cette vie. Le fondement de leur réussite réside dans le désespoir, dans la perception qu'a le prisonnier du fait qu'il est totalement, pour toujours et sans espoir, à la merci de ses geôliers. 
Durant mes années de prison - raconte Pasqualini - j'ai connu un homme qui avait en fait été arrêté par erreur il portait le même nom que la personne recherchée. Au bout de quelques mois, il avait avoué tous les crimes de l'autre. Quand on découvrit la méprise, les autorités de la prison eurent toutes les peines du monde à le persuader de rentrer chez lui. Il se sentait trop coupable pour ça (p. 41).

Il existe en Chine populaire deux sortes d'aveux : l'aveu "pâte dentifrice" (le détenu a besoin d'être pressé de temps à autre, ou bien oublie de continuer à avouer) et l'aveu "eau de robinet" (le prisonnier nécessite une bonne et vigoureuse torsion au départ, mais ensuite tout sort d'un seul coup). Dans les deux cas, le détenu ne dispose d'aucune défense pendant l'interrogatoire; son arrestation est la preuve absolue et indiscutable de sa culpabilité.

Lorsque l'interrogatoire est terminé, le dossier est montré à l'inculpé. On y trouve souvent des dénonciations faites par des collègues, des amis ou toutes sortes de gens qu'on n'a rencontrés qu'une ou deux fois.

Le procès proprement dit est très bref. Le procureur lit l'inculpation officielle et, puisque l'accusé a avoué, aucune défense n'est jugée nécessaire. Le condamné est ensuite transféré au Centre de Transit, où il connaîtra sa sentence. En Chine, on en appelle fort rarement d'une condamnation; l'appel signifie que le prisonnier ne se repent pas de ses crimes et n'a pas accepté l'indulgence du gouvernement; en général, il en découle une punition supplémentaire.

Parmi l'arsenal des peines, il convient de mentionner une invention des communistes chinois : la condamnation à mort avec deux ans de sursis pour l'exécution accompagnée de travaux forcés avec observation de l'effet produit... On n'en viendra à l'exécution du condamné que s'il se conduit mal en prison de façon caractérisée selon des témoignages de ses gardiens. Il va sans dire que ce procédé tend à fabriquer des prisonniers modèles.

III. Les prisons en Chine sont moins des endroits de détention que des écoles où l'on apprend à reconnaître ses fautes.

Les Chinois ont inventé pour leurs prisons les noms les plus poétiques du monde : l'Allée de la Brume sur l'Herbe, le Clair Ruisseau, le Bosquet des Actions Vertueuses, le Précieux Village du Nord, le Lac de l'Enthousiasme Surgissant, etc. Ils ont été également les premiers à saisir l'art de «motiver" les prisonniers, 
grâce au Lao Gai, la Réforme par le Travail. Certes, d'autres pays ont, avant eux, accompli des réalisations particulièrement «remarquables» en ce qui concerne les centres de déportation, les camps de concentration et les prisons-fermes. Ces institutions étaient néanmoins d'une cruauté brutale, sans aucun raffinement, inefficaces en somme, en comparaison de ce que les Chinois ont instauré après la révolution de 1949; le régime de Mao a vite compris que le travail des détenus ne pouvait en aucun cas être productif ou profitable s'il n'était obtenu que par la contrainte ou la torture.

L'histoire du Lao Gai n'est pas seulement l'histoire de Jean Pasqualini, mais aussi, ce qui est beaucoup plus important, celle de millions de Chinois. Une évaluation très réaliste ferait monter le chiffre de candidats à la Réforme par le travail à plus de seize millions de personnes, qui vivent ou ont vécu souvent dans une misère abjecte et désespérante, hantées par la faim, perpétuellement soumises non seulement à l'autorité des gardiens mais surtout à la "surveillance mutuelle» des compagnons de prison, aux propres autoconfessions et aveux pleins de zèle. Sans compter les personnes qui subissent la peine commune de trois ans appelée Lao Jiao, l'éducation par le travail, applicable aux gens qui ont commis des "erreurs» plutôt que des «crimes» et dont le nombre serait égal ou supérieur à celui des individus soumis au Lao Gai.

Sauf des très rares exceptions, l'expérience des camps de travail est totale et permanente; les hommes et les femmes condamnés à la Réforme par le travail passent le reste de leur vie dans les camps, comme prisonniers d'abord, puis, après l'expiration de leur peine, comme "travailleurs libres».

Les camps de travail, en Chine, représentent un contrat à vie. Ils sont trop importants à l'économie nationale pour fonctionner avec du personnel temporaire. Ce sont des détenus qui ont défriché et fait prospérer les vastes étendues stériles de la Mandchourie, lesquelles étaient restées rebelles à toutes les tentatives antérieures, et offrent aujourd'hui encore la seule preuve qu'une ferme d'État de type sovkhozien peut être rentable; ce sont des détenus quí ont été les pionniers de l'industrie plastique chinoise, et qui assurent la production d'un certain nombre de ses plus grandes usines et de ses plus importants centres agricoles; ce sont des détenus qui cultivent le riz même que mange Mao. Pour réussir tout cela, un élément était indispensable : une provision de main-d'oeuvre stable et décidée à travailler dur. En s'assurant cela, les Chinois ont atteint un objectif que même Staline n'avait pas pu réaliser : transformer le travail forcé en une affaire qui 
rapporte. La Chine est sûrement le seul pays au monde à tirer profit de ses prisons (Pasqualini, p. 11-12).

Les "travailleurs libres" mènent une vie analogue à celle des condamnés : même organisation en brigades, même régime carcéral, mêmes tâches, mêmes séances d'étude, mêmes examens idéologiques trimestriels, sans parler de l'adhésion automatique aux normes de la surveillance, de l'accusation et de la dénonciation mutuelles qu'on attend d'eux. Ils jouissent cependant de quelques privilèges, tels la permission de rendre visite à leurs familles deux fois par mois ou le droit d'occuper les premiers rangs lorsqu'il y a projection d'un film dans le camp. Il s'agit, en somme, pour le gouvernement chinois, d'hommes qui ont expié leurs fautes mais qui ont néanmoins commis des crimes et qui doivent "payer" au gouvernement le temps et l'argent que celui-ci a investis pour les amener au niveau technique qu'ils ont atteint.

Quels sont les moyens utilisés par les autorités chinoises pour "motiver» leurs prisonniers? De façon générale, ils découlent de la politique du gouvernement vis-à-vis de ce type d'individus. Les principes suivants, affichés dans toutes les prisons de la Chine, résument bien cette politique : «indulgence à l'égard de ceux qui avouent, sévérité à l'égard de ceux qui résistent; expiation des fautes par l'obtention de mérites; récompenses à ceux qui ont gagné de grands mérites.»

En pratique, cela se traduit par des séances quotidiennes de méditation (durant lesquelles ont doit réfléchir à ses fautes) et d'étude (lecture et discussions orientées à partir d'extraits du Quotidien du Peuple ou des ouvres de Mao); par l'examen idéologique complet qui avait lieu une fois par mois en présence de toute la cellule; par la présence continue d'instructeurs politiques et de propagandistes; par des campagnes de dénonciation, au cours desquelles chacun devait, sans aucune considération de sentimentalité, dénoncer les autres pour ce qu'ils ont dit ou fait contre le gouvernement; par de nombreux discours d'admonestation ayant pour but de demander aux prisonniers de réformer leurs mauvaises pensées et de redevenir des hommes nouveaux; par des punitions très sévères à tous ceux qui refusaient la rééducation par le gouvernement (les mettre aux fers et les jeter au cachot jusqu'à ce qu'ils donnent des signes de repentir); par l'Épreuve.

Cette dernière institution est une invention spécifiquement chinoise, née dans le courant des années trente, qui combine 
l'intimidation, l'humiliation et l'épuisement pur et simple du prisonnier. Il s'agit de sévices intellectuels exercés collectivement contre un seul homme par de nombreux autres, sévices contre lesquels la victime n'a aucun moyen de défense, pas même la vérité. La technique est d'une extrême simplicité : tous les prisonniers réunis sont encouragés à insulter la malheureuse victime, à l'étourdir de leurs cris, voire à la gifler, à lui cracher au visage ou à la battre. Celle-ci ne résiste jamais longtemps, d'autant plus que l'Épreuve ne comporte jamais de limite de temps.

$\mathrm{Au}$ bout de trois ou quatre jours, la victime commence à inventer des fautes qu'elle n'a jamais commises, dans l'espoir qu'un aveu, suffisamment monstrueux lui fera obtenir un sursis. Au bout d'une semaine d'Épreuve, elle est prête à dire et à faire absolument n'importe quoi (p. 65).

Les contrerévolutionnaires et les délinquants politiques étaient d'abord détenus à la Prison de l'Allée de la Brume sur l'Herbe - officiellement le Centre de détention du bureau de la sécurité publique de Pékin. Ils étaient divisés en deux catégories : les personnes ayant un passé contrerévolutionnaire et les contrerévolutionnaires en activité depuis la Libération. Ce Centre, dressé au même endroit où autrefois s'élevait un monastère boudhiste (les Occidentaux ne sont donc pas les seuls à bâtir leurs établissements pénitentiaires sur les restes d'anciens édifices religieux), se composait de plusieurs bâtiments divisés en blocs, le tout entouré d'un mur de brique d'une hauteur d'environ vingt pieds et surmonté de l'inévitable fil barbelé électrifié. Ce Centre aurait été démoli vers les années soixante et remplacé par un nouveau centre d'interrogation baptisé, comme les autres, d'un nom poétique : le Bosquet des Actions Vertueuses.

Après avoir terminé la période d'interrogation et en attendant de comparaître devant le tribunal, d'être condamné ou affecté à une autre prison ou à un autre camp, tout détenu passait ordinairement par le Centre de Transit, situé à Pékin - l'équivalent de nos Centres de classification. De là, il était expédié à un des camps ou prisons du pays. Pasqualini a connu le camp de Ching Ho (Clair Ruisseau), où, même malade, il devait travailler afin de sauver les récoltes de céréales; le Précieux Village du Nord, où étaient envoyées les personnes infirmes ou très malades et qui avait la réputation d'être un camp de la mort; le camp 585, spécialement conçu pour les besoins des malades; le fameux camp de Hsing Kai-Hou (le Lac de l'Enthousiasme Surgissant), situé en Mand- 
chourie, sur des terres de très mauvaise qualité, où étaient internés les gens qui voulaient faire un effort supplémentaire et montrer leur gratitude au gouvernement (les demandes pour aller à ce Camp étaient nombreuses, en raison de sa meilleure nourriture et de l'absence de normes de rendement; les détenus y ont créé des champs de légumes, un verger d'arbres fruitiers, une usine de conserves de soja et exploité la viande de porc et autres animaux); la prison de Langxiang (le Village Vertueux), une usine de machines électroniques de la capitale, construite à toute hâte dans la banlieue de Pékin afin de construire des ustensiles en aluminium (essentiellement, des marmites et des casseroles bon marché); finalement, la prison modèle de Pékin, connue officiellement sous le nom de Complexe Industriel du Clair Ruisseau, sans barreaux aux fenêtres ni verrous aux portes, où la nourriture n'était pas rationnée et le travail était humain, digne et rémunéré, en somme, un endroit convenable où les détenus se sentaient vraiment heureux (cette prison est encore aujourd'hui une des meilleures attractions touristiques de Pékin et a été conçue pour témoigner de l'attitude progressiste de la Chine en matière de politique pénitentiaire).

Entre 1957 et 1964, un des problèmes les plus dramatiques des prisons et camps de travail de Mao était la nourriture, ou plutốt le manque de nourriture, qui était admirablement étudié. Les détenus recevaient assez pour les maintenir en vie, mais jamais assez pour leur faire oublier leur faim. Durant les quinze mois passés par Pasqualini au Centre d'interrogation, il mangea une seule fois du riz, jamais de la viande. Les détenus prenaient deux repas par jour, qui se composaient d'une très mince galette de pain de maïs (environ cent grammes), un morceau de légume salé (une tranche de navet bouilli d'à peu près un centimètre et demi d'épaisseur) et un bol de gruau de céréales, qui contenait surtout de l'eau. La nourriture constituait une véritable obsession pour tous les prisonniers, qui inventaient des gestes rituels et quasi religieux pour entourer sa présentation et sa consommation à mesure qu'elle se faisait de plus en plus rare : mastication lente, réduction des aliments solides à des morceaux minuscules. Lors des périodes de famine, les prisonniers chinois ont mangé des vers trouvés dans les bouses des vaches et des bœufs, des particules de maïs non digérés trouvés dans le crottin des chevaux des gardes. Les autorités, de leur côté, ont fait manger aux détenus de la pâte à papier (ce qui provoqua une constipation collective, au cours de laquelle plusieurs personnes âgées ou faibles sont mortes en 
essayant de toutes leurs forces d'aller à la selle) et du plancton des marais. Après quelques mois d'un régime semblable, le ventre des prisonniers était complètement aplati, les ongles cassaient au moindre effort, la peau se détachait du corps et les cheveux tombaient.

Très souvent, la nourriture du prisonnier dépendait de sa production au travail. C'est ainsi qu'au Centre de Transit, où les détenus devaient plier les pages des livres et les ficeler en paquets, le but à atteindre fixé par le gouvernement était de 6000 feuilles par jour alors que la production moyenne était de 4500 feuilles et le rendement normal pour un débutant de 3000 ; les rations étaient divisées en catégories qui correspondaient au degré d'adresse atteint par le prisonnier.

En plus du pliage de feuilles de livres, le travail effectué dans les camps chinois touchait la construction (un bataillon formé des 400 ou 500 architectes, géomètres, ingénieurs, menuisiers, maçons, etc., condamnés à perpétuité parcourait toute la Chine en construisant des prisons et en perfectionnant sans cesse leurs techniques afin de gagner des points de mérite), la traduction (moyenne obligatoire de 4000 mots par jour), la production de ceintures en plastique et de bas en nylon et, surtout, les travaux agricoles (préparer des terres incultes pour l'exploitation, effectuer des récoltes, voire ramasser les excréments des cochons afin de les utiliser comme engrais). En fait, le but poursuivi par le gouvernement était d'avoir une main-d'œuvre d'esclaves à bon marché, qui travaillait pour atteindre la plus forte production possible. Pour ce faire, tous les moyens semblaient bons, y compris celui consistant à encourager les équipes à se lancer des défis et de récompenser les gagnants par de la nourriture supplémentaire.

Le problème de la sexualité dans les prisons, pas encore réglé de façon satisfaisante dans les établissements pénitentiaires d'Occident, ne semble pas causer de grands soucis aux autorités chinoises et cela se comprend aisément. Outre le fait que les Chinois sont en général plus réservés que les Occidentaux en ce qui concerne les questions sexuelles, la combinaison de la faim perpétuelle, du manque de vitamines et de l'épuisement provoqué par le dur travail physique amenait les prisonniers à un tel état de faiblesse qu'ils étaient tous virtuellement impuissants. Sans parler des peines très sévères encourues, comme nous l'avons déjà vu, par tous ceux qui se rendaient coupables de délits à caractère sexuel. 
En général, la vie quotidienne dans une prison chinoise ressemble, à quelques nuances près, à celle que l'on peut mener dans n'importe quelle autre prison du monde. Les prisonniers ont le droit d'écrire à l'extérieur et de recevoir des visites. Les rapports entre eux sont positifs, malgré le fait qu'à l'occasion de certains événements (campagnes de dénonciation, l'épreuve) la prison se transforme un une jungle féroce. Lisons, à cet égard, Pasqualini :

Ce qui me sidérait, c'était la façon dont ils pouvaient agir comme si rien ne s'était passé. Finalement, au bout de quelques jours, je parvins à comprendre. À quoi bon leur garder rancune. Ils avaient été contraints de me faire subir l'épreuve. Ils n'avaient pas d'autre choix. C'était simplement un fait de la vie chinoise. S'ils avaient refusé de se joindre au groupe, cela aurait signifié une mauvaise note sur leur dossier et, probablement, à la fin, une Épreuve pour eux. Il n'y avait pas d'autre moyen de s'en sortir que de suivre le mouvement, de faire ce qu'on leur demandait et d'en finir aussi vite que possible, puis d'oublier tout ce qui s'était passé (p. 141).

Il arrivait aussi que, chaque membre d'une cellule ou d'une équipe devant dénoncer régulièrement les autres, ceux-ci se mettent d'accord pour désigner d'avance le coupable de la journée ce qui assurait un certain équilibre à la fin du mois en ce qui concerne les «mauvaises notes» de chaque membre. D'après Pasqualini, c'est dans les prisons et les camps que les notions d'amitié et de liberté se sont développées au plus haut point en Chine.

Les rapports avec les gardiens pouvaient également adopter des formes déroutantes pour un esprit occidental. En premier lieu, les surveillants des camps étaient des ex-prisonniers ayant atteint un niveau idéologique suffisant. Ils étaient ensuite des instructeurs politiques, qui participaient pleinement à l'œuvre générale de "réhabilitation" du détenu. Un certain ritualisme accompagnait par ailleurs les relations gardiens-prisonniers en cas d'infractions commises par ceux-ci. Un prisonnier, par exemple, perdait la face s'il cédait trop facilement aux pressions du gardien et, chose surprenante, le gardien aussi; il fallait permettre au détenu de développer jusqu'au bout la logique de son jeu, de la même façon que le surveillant devait, utilisant la dialectique communiste, le convaincre lentement mais sûrement, qu'il avait tort.

L'expérience de la prison, et peut-être même le simple fait d'avoir été inculpé pour des activités contre-révolutionnaires, en- 
traînent, en Chine comme ailleurs, des réactions de stigmatisation fort accentuées. Pasqualini cite le cas de certains prisonniers libérés qui, étant retournés chez eux et fait l'objet du mépris et des sarcasmes de la part des propres membres de leurs familles, sont revenus à la prison - leur seule vraie maison.

Le Livre de Pasqualini, même s'il ne représente que le témoignage d'une seule personne et ne se réfère qu'à une courte période de l'histoire chinoise, vieille déjà d'une dizaine d'années, demeure cependant d'actualité car il décrit un univers qui continue à faire partie de la construction de la Chine nouvelle. Il confirme le caractère implacable de la logique du système concentrationnaire, qui est le même partout - ainsi que l'a démontré Soljenitzyne - et témoigne de procédés collectifs de lavage de cerveau semblables à ceux que les procès de Moscou ou de Budapest nous avaient fait connaître. Il montre finalement les efforts déployés par un système oppressif en vue d'anéantir l'homme. 